\title{
EBSD Surface Topography Determination in a Martensitic Au-Cu-Zn Alloy
}

\author{
M. Chapman ${ }^{1}$, P. Callahan ${ }^{2}$, M. De Graef ${ }^{1}$ \\ ${ }^{1}$ Dept. of Materials Science and Engineering, Carnegie Mellon Univ., Pittsburgh PA 15213, USA \\ ${ }^{2}$ Dept. of Materials Science, University of California at Santa Barbara, Santa Barbara CA 93106, USA
}

$\mathrm{Au}_{30} \mathrm{Cu}_{25} \mathrm{Zn}_{45}$ is a shape memory alloy with a composition that has been optimized to achieve a low hysteresis martensitic transformation, by increasing the degree of compatibility between the cubic austenite and the monoclinic martensite lattices [1]. This degree of lattice match has profound consequences for the multi-variant microstructure in that there is now no elastic energy penalty associated with the presence of variant-variant boundaries; this, in turn, can give rise to interesting curved martensite-austenite boundaries as well as the fact that the microstructure becomes completely irreproducible from one thermal cycle to the next. In our work, we attempt to verify a mathematical model [1] for the variant-variant geometries that occur during the transformation in $\mathrm{Au}_{30} \mathrm{Cu}_{25} \mathrm{Zn}_{45}$; in particular, we are interested in determining the complete transformation strain for each variant, including the out-of-plane component at the sample surface. Such a topographic measurement is made difficult because (a) the transformation temperature of the material is $-50^{\circ} \mathrm{C}$ and (b) as stated above, every time the sample is transformed the martensite variant arrangement is completely different. Since crystallographic data needs to be collected along with the topography data to determine strain states and thus confirm the model, the topographic data needs to be collected simultaenously with the EBSD data. A novel technique that uses the background intensity of the EBSD patterns was used to achieve this result.

The Backscatter Electron Surface Topography (BEST) method uses the EBSD detector and is based on the fact that, on average, backscattered electrons exhibit nearly specular reflection with respect to the surface of the sample; this can be modeled and confirmed by Monte Carlo (MC) simulations. For truly specular reflection, and assuming a spherical sample surface, the incident electron beam, the local surface normal, and the direction of maximum outgoing EBSD background intensity will all lie in a single plane. MC simulations can predict the deviation from the case of specular "reflection," which depends on the sample average atomic number, the microscope accelerating voltage, and the electron beam inclination angle, and can be expressed as a simple power series in the incidence angle. MC simulations were carried out for $\mathrm{Au}_{30} \mathrm{Cu}_{25} \mathrm{Zn}_{45}$ to determine the magnitude of specular deviation.

An EBSD scan of martensitic $\mathrm{Au}_{30} \mathrm{Cu}_{25} \mathrm{Zn}_{45}$ is shown in Fig. 1a (Each pixel represents one EBSD pattern). Each $320 \times 240$ pixel EBSD pattern is fitted with a 2-D Gaussian distribution to determine the location of the maximum peak of the background intensity. This location is used to determine the relative shift of the peak from the center of the detector. The vertical component of the shift is depicted in Fig. 1b; green indicates a peak position 50 pixels above and black 13 pixels below the horizontal center line. Fig. 1c shows the horizontal component of the peak shift; red indicates a peak position 9 pixels left and black 6 pixels right of the vertical center line. The locations of the background peaks for the entire scan were binned and six predominant bins were identified. These six bins were randomly assigned colors and the resulting color map is shown in Fig. 1d. The background peak locations, along with the sample and experimental geometries allow for the calculation of the local surface normal. The negative gradient of the surface height function, $\hat{\mathbf{n}}(x, y)=-\nabla z(x, y)$, represents the local surface normal with components $n_{x}=-\partial_{x} z$ and $n_{y}=-\partial_{y} z$. The surface height function, $z(x, y)$, can then be retrieved by means of Fourier transforms to invert the gradient operator. The resulting surface height function, exaggerated in the vertical direction, is shown Fig. 2. 


\section{References}

[1] Y Song, X Chen, and RD James, Nature 502 (2013), p. 85.

[2] PG Callahan and M DeGraef, Microscopy and MicroAnalysis 19 2013, p.1255-1265.

[3] Research supported by the Air Force Office of Scientific Research, MURI contract \# FA9550-12-1-0458.

a)

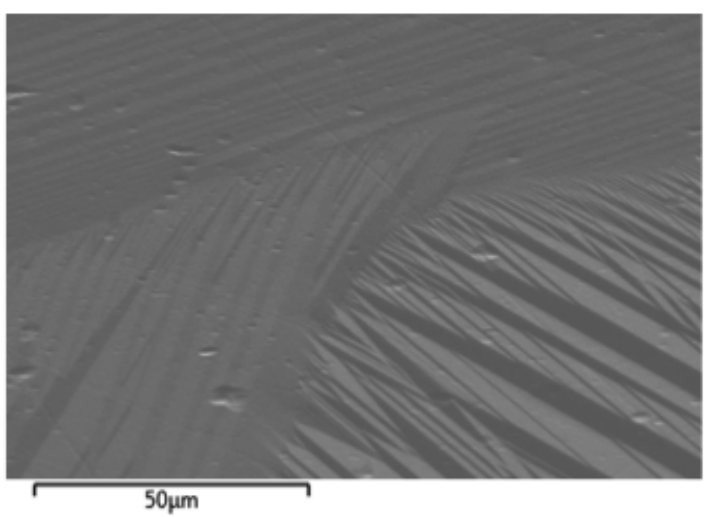

c)

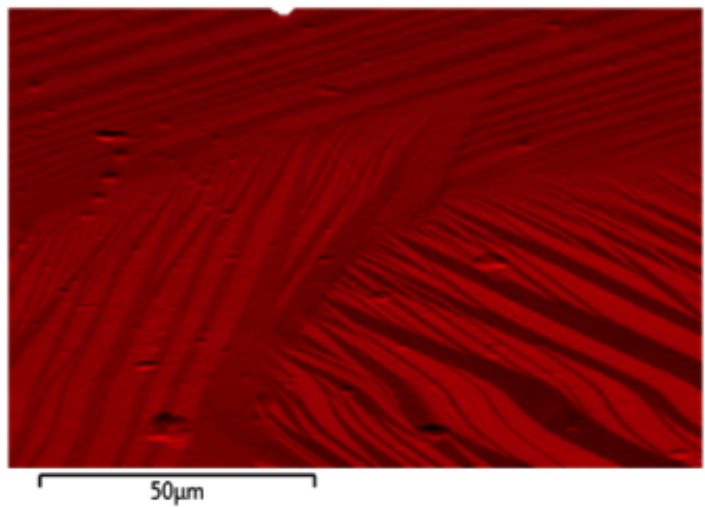

b)

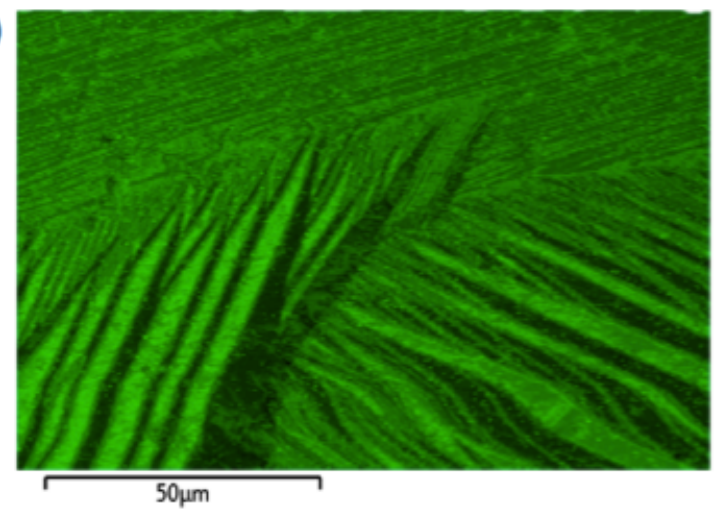

d)

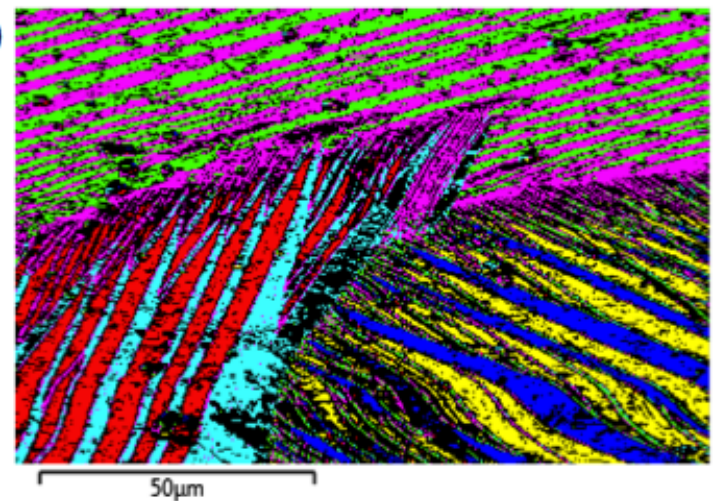

Figure 1: a) Secondary electron micrograph of martensitic $\mathrm{Au}_{30} \mathrm{Cu}_{25} \mathrm{Zn}_{45}$; b) vertical position of the background intensity peak on the EBSD pattern (see text for details); c) horizontal position of the background intensity peak; d) colorized map of the six clusters in the peak locations, indicating six different groups of sample surface inclinations that correspond to the individual martensite variants.

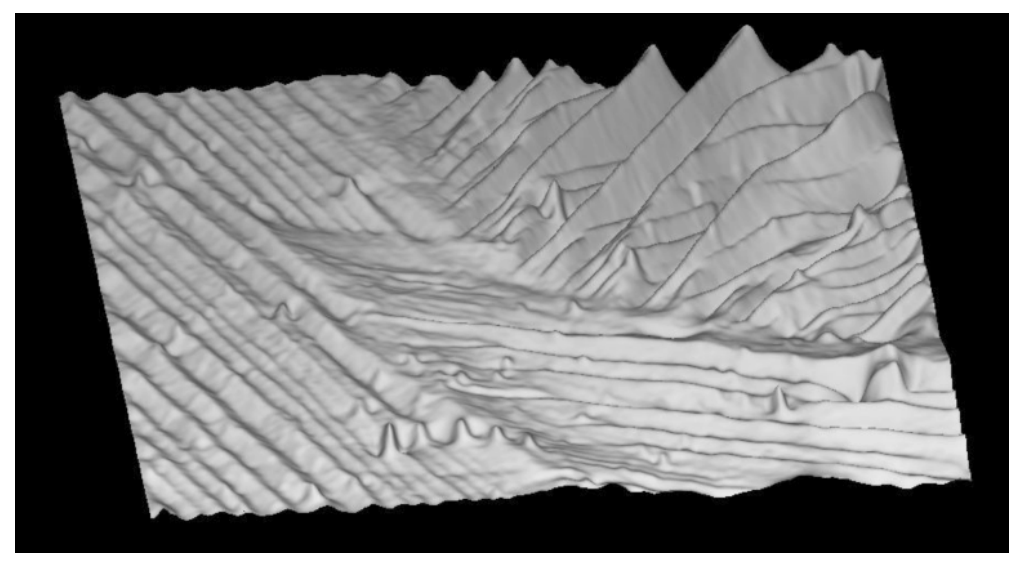

Figure 2: Rendering of the reconstructed three dimensional surface of the martensitic $\mathrm{Au}_{30} \mathrm{Cu}_{25} \mathrm{Zn}_{45}$ alloy using BEST; the vertical dimension is exaggerated to highlight the topography. The surface is rotated $90^{\circ}$ counterclockwise with respect to the images in Fig. 1. 